\title{
Onkologische Therapien - was sind patientenrelevante Endpunkte?
}

\author{
Michael Ehmann ${ }^{\mathrm{a}} \quad$ Martin Härter ${ }^{\mathrm{b}} \quad$ Ralf Rambach $^{\mathrm{c}} \quad$ Eva Winkler $^{\mathrm{d}}$ \\ a Selbsthilfe für Lungenkrebs, Landesverband Baden-Württemberg, Thoraxklinik am Universitätsklinikum Heidelberg, \\ ${ }^{\mathrm{b}}$ Institut und Poliklinik für Medizinische Psychologie, Universitätsklinikum Hamburg-Eppendorf, \\ ${ }^{c}$ Deutsche Leukämie- und Lymphom-Hilfe e.V., Bonn, \\ ${ }^{\mathrm{d}}$ Abteilung Medizinische Onkologie, Nationales Centrum für Tumorerkrankungen, Heidelberg, Deutschland
}

\section{Teil 1: Bedürfnisse der Patienten integrieren Verantwortliche Autoren: Martin Härter, Hamburg, Eva Winkler, Heidelberg}

\section{Entscheidungspraxis in der Klinik: Praktische Urteilskraft- Institutionelle Ethik}

Therapieempfehlungen werden in der Onkologie zunehmend in interdisziplinär zusammengesetzten Tumorboards unter Berücksichtigung der evidenzbasierten Datenlage, der vorhandenen klinischen Erfahrung und der Abwägung von Nutzen und Belastung für den Patienten besprochen. Zusätzlich sollten in die definitive Therapieentscheidung die Vorstellungen, Präferenzen bzw. Wünsche des betroffenen Patienten einfließen. Die Empfehlungen des Tumorboards müssen daher mit dem Patienten besprochen und Vor- bzw. Nachteile verschiedener Therapieoptionen vor dem Hintergrund der medizinischen und persönlichen Situation dargelegt und diskutiert werden. Gerade bei weit fortgeschrittenen Erkrankungen, wenn keine Heilungsoption mehr besteht, können unter Nutzen-Risiko-Gesichtspunkten ethisch schwierige Entscheidungen zu treffen sein. Hier kommt es vor, dass die Vorstellungen des Patienten hinsichtlich des Behandlungsziels nicht mit den Empfehlungen des Arztes bzw. Tumorboards deckungsgleich sind. Die Frage ist, wie damit im klinischen Alltag umzugehen ist.

\section{Ethisch schwierige Therapieentscheidungen}

Tatsächlich ist wenig über die konkrete Entscheidungspraxis im klinischen Alltag bekannt und inwieweit die Präferenzen der Patienten erfragt und berücksichtigt werden. Grundsätzlich lassen sich in der palliativen Therapiesituation bei Patienten mit fortgeschrittener Tumorerkrankung und einer verbleibenden medianen Lebenserwartung von 6-12 Monaten
3 Patientengruppen unterscheiden [1-3]: Etwa ein Drittel dieser Patienten präferiert eine rein symptomatische, auf die Lebensqualität ausgerichtete Behandlung, ein Drittel wünscht bis zum Lebensende eine maximale Therapie und ein Drittel ist zunächst unentschieden, präferiert aber mit fortschreitender Erkrankung eine primär an der Lebensqualität orientierte Behandlung. Zwischen den Patientengruppen zeigen sich Unterschiede bei der Krankheitsbewältigung. So scheinen Patienten, die sehr lange an einer maximalen Therapie festhalten, häufiger eine unrealistische Prognoseeinschätzung zu haben als jene, die sich für eine symptomorientierte Behandlung entscheiden [1, 4].

Die Diskrepanz zwischen dem Therapieziel des Arztes und dem des Patienten kann auf einer faktischen oder einer normativen Differenz beruhen: (1) Die faktische Differenz basiert darauf, dass der Patient seine Krankheitssituation nicht realistisch sieht [4, 5]. (2) Eine normative Differenz besteht, wenn der Patient die Situation zwar realistisch sieht, sie aber für sich anders bewertet als der Arzt. Dies ist gerade bei schwerkranken Patienten häufiger der Fall, die eher bereit sind, belastende Therapien zu ertragen als Gesunde [6, 7].

Aus Sicht des Behandlers stellt sich in solchen Situationen die Frage, ob ein Therapieverzicht gegen den Patientenwillen ethisch begründbar ist bzw. ob er intensiver behandeln sollte, als er es für sinnvoll hält, weil der Patient dies wünscht. Eine weitere Frage ist, ob es legitim ist, dem Patienten eine intensivere Therapie gar nicht anzubieten, weil sie aus ärztlicher Sicht nicht infrage kommt. Klassischerweise stützt sich die Therapieentscheidung auf die medizinische Indikation und den Patientenwillen. Lehnt der Patient eine Behandlung ab, darf diese nicht durchgeführt werden. Die Frage bleibt, ob der Arzt eine Therapie anbieten muss, die er nicht für indiziert hält bzw. für die es keine ausreichenden Wirknachweise gibt.

Aus ethischer Sicht ist es wichtig, dass die Therapieent-

\section{KARGER}

Fax +497614520714

Information@Karger.com

www.karger.com (c) 2013 S. Karger GmbH, Freiburg

0378-584X/13/3618-0005\$38.00/0 
scheidung transparent ist und dass sie neben den medizinischen Kriterien auch die Sichtweise des Patienten einschließt. Die Wirksamkeit beurteilt der Arzt bzw. das Ärzteteam im Tumorboard. Das Nutzen/Belastung-Verhältnis bewerten Arzt und Patient. Hier kann es zu Differenzen kommen $[8,9]$.

\section{Integration in den klinischen Alltag}

Wie lassen sich die dargelegten Überlegungen aus ethischer Sicht in den klinischen Alltag integrieren, um eine gute Entscheidungspraxis für den Einzelfall zu gewährleisten? Voraussetzung ist aus ethischer Sicht die praktische Urteilskraft, sprich die Fähigkeit zu angemessenem Handeln im konkreten Einzelfall unter Berücksichtigung aller für die Situation relevanten Faktoren, der individuellen Handlungsziele und ethischen Wertmaßstäbe. In der Literatur wurde in den letzten Jahren immer häufiger betont, wie wichtig es ist, ethische Wertmaßstäbe klinikintern zu diskutieren und festzulegen [10, 11].

Medizin wird heute im Team und eingebunden in einen institutionellen Rahmen praktiziert. So sitzen beispielsweise im Tumorboard Vertreter verschiedener Fachdisziplinen. Bei strittigen Bewertungsfragen bietet es sich an, ein Forum der gemeinsamen Meinungs- und Konsensbildung zu haben, wie z.B. eine klinische Ethikberatung. Solche klinischen Ethikberatungsdienste sind eine wichtige institutionelle Antwort auf ethische Konflikte und werden auch von der zentralen Ethikkommission der Deutschen Ärztekammer propagiert [12]. In den USA und zunehmend auch in Deutschland haben viele größere Krankenhäuser sogenannte klinische Ethikkomitees. Aufgabe der Ethikkomitees ist es, die Konsensbildung im Team zu unterstützen, für wertbasierte Entscheidungen zu sensibilisieren und Leitlinien zu verfassen, an denen sich Entscheidungsträger orientieren können, wenn es um ethisch schwierige Situationen geht [13-15]. Um die Patientenversorgung entscheidend zu verbessern, muss eine Ethikberatung initiativ und nicht nur reaktiv agieren. Vertreter des Ethikkomitees sollten in die jeweilige Institution integriert und bezüglich ihrer Tätigkeit rechenschaftspflichtig sein [16].

\section{Anspruch und Wirklichkeit von «Shared Decision Making»}

Neben der ethischen Perspektive bei der Therapieentscheidung stellt sich im klinischen Alltag die Frage, welche Entscheidungsmodelle zur Verfügung stehen, um die Präferenzen der Patienten adäquat zu berücksichtigen und die normative und faktische Ebene im individuellen Arzt-Patienten-Gespräch auf einen gemeinsamen Nenner zu bringen. Das paternalistische - aus Patientensicht passive - Entscheidungsmodell, bei dem der Arzt bzw. das Tumorboard die Therapieentscheidung ohne Mitwirken des Patienten trifft, wird von vielen Patienten nicht mehr akzeptiert. Viele Patienten wollen in die Therapieentscheidung eingebunden sein. Dieses Modell der sogenannten partizipativen Entscheidungsfindung (PEF) zwischen Arzt und Patient basiert auf einem Interaktionsprozess mit gleichberechtigten Partnern, um eine gemeinsam verantwortete Übereinkunft zu erzielen [17, 18]. Grundvoraussetzung ist, dass der Arzt den Patienten als Person mit seiner Erkrankung, seiner Krankheitserfahrung und seinen Präferenzen hinsichtlich der Behandlung ernst nimmt. Ziel ist eine stabile therapeutische Allianz zwischen Arzt und Patient, die insbesondere bei kritischen Therapieentscheidungen notwendig ist.

Bei der PEF stehen Wissen und Erfahrung des Arztes sowie seine Werte und Einstellung den individuellen Wünschen und Werten des Patienten gegenüber. Beides sollte in Einklang gebracht werden, was in der Onkologie nicht selten zu schwierigen Abwägungsprozessen führen kann. Je schwieriger die Entscheidung nach rein medizinischen Kriterien zu treffen ist, desto wichtiger ist die persönliche Präferenz des Patienten [18].

\section{PEF ist «Shared Decision Making»}

Der Therapievorschlag des Tumorboards formuliert eine Empfehlung, die mit dem Patienten besprochen wird. Das beinhaltet immer auch die Option, dieser Empfehlung nicht zu folgen. Damit Arzt und Patient dies gemeinsam und aus einer Position der Gleichwertigkeit («Equipoise») entscheiden können, muss der Patient gut aufgeklärt sein, die infrage kommenden Behandlungsmöglichkeiten und die jeweiligen Vorund Nachteile bzw. Risiken kennen und bezüglich der Konsequenzen einschätzen können. Der Arzt muss in der Lage sein, Verständnis für die Situation des Patienten zu entwickeln und seine Befürchtungen und Erwartungen aktiv kennenzulernen. Für die Entscheidungsfindung sollte im Vorfeld die Rollenpräferenz geklärt worden sein. Nicht jeder Patient möchte die Entscheidung treffen; manche Patienten delegieren diese weitgehend an den Arzt [19].

Wichtig ist, dass der Patient dem Gespräch inhaltlich folgen kann und die medizinischen Hintergründe versteht. Sprache und Inhalte müssen folglich an das Wissensniveau des Patienten angepasst werden. Wichtig ist auch, dem Patienten Zeit für seine Entscheidung einzuräumen. Schriftliche Materialien und grafische oder visuelle Informationen zu den Behandlungsmöglichkeiten (sogenannte medizinische Entscheidungshilfen) erleichtern die Gesprächsführung und unterstützen den Informationsfluss und das Verständnis des Patienten. Bei entsprechenden Informationsmaterialien besteht in Deutschland noch erheblicher Nachbesserungsbedarf (die in Deutschland verfügbaren Entscheidungshilfen können unter $w w w$.patient-als-partner.de abgerufen werden).

Nachbesserungsbedarf scheint auch bei der Berücksichtigung von Patientenwünschen im Rahmen der Therapieentscheidung zu bestehen. Eine Studie bei 137 Patientinnen mit primärem Mammakarzinom zeigt beispielsweise, dass im klinischen Alltag nicht alle Patientinnen entsprechend ihren Vorstellungen an der Entscheidungsfindung partizipieren. Darüber hinaus weist die Untersuchung darauf hin, wie wichtig es ist, die Wünsche der Patienten bei der Entscheidungsfindung zu berücksichtigen: Jene Patientinnen, die ihren Vorstellungen 
entsprechend an der Therapieentscheidung beteiligt waren, waren im späteren Behandlungsverlauf zufriedener, fühlten sich weniger belastet und hatten seltener psychische Beschwerden bzw. Symptome. Dieser Befund wurde unabhängig davon beobachtet, für welches Entscheidungsmodell sich die Patientinnen entschieden hatten [20, 21]. Die Untersuchung verdeutlicht, dass es darum geht, die richtige Entscheidungsform für den einzelnen Patienten zu finden.

Die gezielte Umsetzung einer PEF führt bei Ärzten und Patienten zu positiven Effekten: Die Ärzte sind selbst mit dem Patientenkontakt zufriedener, sie ziehen mehr Behandlungsoptionen in Betracht und stellen fest, dass sich die Konsultationszeit nicht verlängert. Bei den Patienten führt die PEF unter anderem zu einer höheren Zufriedenheit mit dem Arztkontakt und der Therapieentscheidung sowie zu einer besseren Krankheitsbewältigung und korrekteren Risikowahrnehmung [22, 23].

Die PEF ist eine Zielvorgabe, die auch im Rahmen des Nationalen Krebsplans formuliert wurde. Das Erfassen der Patientenbeteiligung wird dort als Qualitätsindikator einer qualitativ hochwertigen, umfassenden onkologischen Versorgung avisiert. Es geht darüber hinaus darum, Wirkungs- und Nutzungsbelege zum Ausbau von Patienteninformationen zu sammeln und spezifische medizinische Entscheidungshilfen und Interventionen zu erarbeiten. Trainingsmodule im Sinne eines gezielten Gesprächstrainings sollen verstärkt in die medizinischen und pflegerischen Curricula integriert werden.

\section{Teil 2: Was ist wichtig aus der Sicht der Betroffe- nen und Angehörigen? \\ Verantwortlicher Autor: Michael Ehmann, Heidelberg}

Patienten mit Lungenkarzinom befinden sich bei Diagnosestellung meist bereits im fortgeschrittenen Stadium mit nur noch begrenzten Heilungschancen. Die verbleibende Lebenszeit ist meist sehr begrenzt. Der patientenrelevante Endpunkt bei Lungenkrebskranken ist in der Regel die Begleitung bis zum Tod. Aus Sicht der Betroffenen und der Angehörigen geht es ganz wesentlich um das «Wie» dieser Begleitung. Vielen Patienten ist ein ganzheitlicher Weg wichtig, der aber nur selten beschritten wird - sei es, weil es der behandelnde Arzt nicht gelernt hat, sei es, dass der Druck des Gesundheitssystems zu groß ist.

Im klinischen Alltag steht oft wenig Zeit zur Verfügung, um sich mit dem Patienten als Person intensiver auseinanderzusetzen und ihn so auch bei der Krankheitsbewältigung zu unterstützen. Der Arzt und Philosoph Karl Jaspers kritisierte bereits 1946 sowohl den «Rigorismus» als auch den «Paternalismus» im ärztlichen Handeln und leitete daraus das Modell der existenziellen Kommunikation ab [24]. Diese meint in der Medizin die «Selbstverständlichkeit, dass der Arzt dem Patienten von Mensch zu Mensch begegnet, ihn also nicht als Fall behandelt, sondern in ihm ein Individuum sieht» [25].
Neben dem Zeitfaktor sind für die Patienten Sprache und Gesprächsinhalte wichtig: Gesprächsinhalte, die nicht hilfreich sind, sollten vermieden werden, z.B. «Wir versuchen, dass der Krebs zum Stillstand kommt.» Es empfiehlt sich, im Gespräch mit dem Patienten nicht nachvollziehbare Begriffe zu vermeiden, wie z.B. «personalisierte Medizin», die bei Patienten auch $\mathrm{zu}$ falschen Vorstellungen führen können. Bei wichtigen Fragen sollte der Arzt eine klare Haltung einnehmen. Spricht der Patient alternative Substanzen wie z.B. Mistelpräparate an, ist eine ehrliche, aber konstruktive Auseinandersetzung wichtig. Vielen Patienten tut es psychisch gut, etwas für sich zu tun. Optional bietet sich daher an, Alternativen vorzuschlagen, wie z.B. körperliche Bewegung. Nicht zu unterschätzen sind die Nebenwirkungen einer onkologischen Behandlung. Ein gutes Nebenwirkungsmanagement ist obligat. Temel et al. [5] konnten bei Patienten mit nichtkleinzelligem Lungenkarzinom (NSCLC) zeigen, dass sich unter einer gut geführten palliativen Therapie nicht nur die Lebensqualität der Patienten bessert, sondern sich auch die Lebenszeit verlängern lässt.

Patienten und Angehörige kritisieren außerdem, dass Selbsthilfegruppen für Lungenkarzinom-Patienten bundesweit oft schlecht organisiert und vernetzt sind. Von unabhängigen Institutionen wie der Deutschen Krebsgesellschaft, der Deutschen Krebshilfe oder den regionalen Krebsgesellschaften fühlen sich die Lungenkarzinom-Patienten nur unzulänglich unterstützt.

\section{Teil 3: Patientenrelevanter Nutzen aus Sicht des AMNOG \\ Verantwortlicher Autor: Ralf Rambach, Bonn}

Das Arzneimittelmarktneuordnungsgesetz (AMNOG) schreibt vor, dass neu zugelassene Medikamente vom Gemeinsamen Bundesausschuss (G-BA) hinsichtlich ihres Zusatznutzens bewertet werden. Dies geschieht anhand operationalisierter Werte, die ins Verhältnis gesetzt und in der Regel anhand patientenrelevanter Endpunkte gewertet werden. In erster Linie sind dies Mortalität, Morbidität und Lebensqualität der Patienten. Der G-BA besteht aus drei Handlungsebenen: dem Plenum, das den definitiven Beschluss fasst, sowie den Unterausschüssen, die die Beschlussempfehlungen auf der Grundlage der Arbeit der Arbeitsgruppen vorbereiten. Arbeitsgruppe und Unterausschuss sind weitgehend personenidentisch und unterscheiden sich de facto nur in der Arbeitsebene. Die «Frühe Nutzenbewertung» nach $\S 35$ a SGBV erfolgt vorbereitend durch die Arbeitsgruppe $\S 35 \mathrm{a}(\mathrm{AG} \S 35 \mathrm{a})$ im Unterausschuss «Arzneimittel» (UA-AM).

Die Patientenvertreter sind in allen Gremien mit beratend tätig. Sie haben ein Antragsrecht, aber kein Stimmrecht. Ihr Votum genießt gleichwohl einen hohen Stellenwert. Eine zweite Form der Patientenbeteiligung ist bei der Dossier- 
bewertung durch das Institut für Qualität und Wirtschaftlichkeit im Gesundheitswesen (IQWiG) vorgesehen, das einen Patientenfragebogen entwickelt hat, der den Selbsthilfeorganisationen die Möglichkeit der Stellungnahme bietet.

\section{Patientenbeteiligung bei der frühen Nutzenbewertung}

Nach Markteintritt - Zulassung oder positiver Stellungnahme der Europäischen Arzneimittel-Agentur (EMA) - informieren die ständigen Patientenvertreter bereits die relevanten Selbsthilfeverbände und suchen geeignete Patienten, die ausreichend Erfahrung mit dem Krankheitsbild, gegebenenfalls sogar mit dem neuen Medikament haben und bereit sind, als sogenannte fachbezogene Patientenvertreter ihre Meinung zur Nutzenbewertung einzubringen. Im nächsten Schritt bearbeitet der UA-AM das vom pharmazeutischen Unternehmer erstellte Dossier zur Nutzenbewertung des Medikamentes nach $\S 35 \mathrm{a}$ SGBV. Dies geschieht unter Mitberatung der ständigen und der fachbezogenen Patientenvertreter. Das erarbeitete Dossier wird parallel vom IQWiG bewertet. Hier sind die fachbezogenen Patientenvertreter über den vom IQWiG entwickelten Patientenfragebogen eingebunden. Auf der Grundlage der Ergebnisse der AG $\S 35$ a erarbeitet der UA-AM eine Beschlussempfehlung für das Plenum, der das Plenum meist folgt. Bei dissenter Empfehlung entscheidet das Plenum per Abstimmung der drei Bänke.
Beschlüsse, denen die Patientenvertreter nicht zustimmen, werden in der Regel erneut verhandelt. Die Problematik der Entscheidung über die Nutzenbewertung bzw. den Zusatznutzen liegt in der Gewichtung von Wirkung und Nebenwirkungen. Problematisch sind Medikamente mit hoher Wirkung, aber auch ausgeprägten Nebenwirkungen sowie weniger wirksame Medikamente mit nur geringen Nebenwirkungen, wo der Zusatznutzen über die gute Verträglichkeit generiert werden soll. Im klinischen Alltag ist in solchen Situationen die Nutzen-Risiko-Bewertung im Einzelfall entscheidend. Solche Einzelfallentscheidungen kann der G-BA nicht treffen. Bei der Nutzenbewertung eines Medikamentes geht es um formalistische Entscheidungen. Aus Patientensicht soll sichergestellt sein, dass auch Medikamente auf den Markt kommen, die unter besonderer Berücksichtigung des Einzelfalls eingesetzt werden können. Um zukünftig eine bessere Grundlage für die Nutzenbewertung zu schaffen, sind verbindliche Voraussetzungen bzw. Standards für kontrollierte klinische Studien notwendig, mit denen sich patientenrelevante Studienendpunkte definieren und belegen lassen.

\section{Disclosure Statement}

Die Autoren waren Referenten beim «Dresdner Interdisziplinäres Gespräch 2013», das von Roche Pharma AG unterstützt wurde.

\section{Literatur}

1 Winkler EC, Reiter-Theil S, Lange-Rieß D, et al.: Patient involvement in decisions to limit treatment: the crucial role of agreement between physician and patient. J Clin Oncol 2009;27:2225-2230.

$\longrightarrow 2$ Voogt E, van der Heide A, Rietjens JA, et al.: Attitudes of patients with incurable cancer toward medical treatment in the late phase of life. J Clin Oncol 2005;23:2012-2019.

>3 Stiggelbout AM, de Haes JC, Kiebert GM, et al.: Tradeoffs between quality and quantity of life: development of the Questionnaire for Cancer Patient Attitudes. Med Decis Making 1996;16:184-192.

4 Temel JS, Greer JA, Pire WF, et al.: Effect of early palliative care on chemotherapy use and end-of-life care in patients with metastatic non-small-cell lung cancer. J Clin Oncol 2012;30:394-400.

5 Temel JS, Greer JA, Muzikansky A, et al.: Early palliative care for patients with metastatatic nonsmall-cell lung cancer. N Engl J Med 2010;363:733742 .

6 Hancock K, Clayton JM, Parker SM, et al.: Truthtelling in discussing prognosis in advanced life-limiting illnesses: a systematic review. Pall Med 2007; 21:507-517.

7 Weeks JC, Cook F, O'Day SJ, et al.: Relationship between cancer patients' predictions of prognosis and their treatment preferences. JAMA 1998;279: 1709-1714.

$>8$ Winkler E et al.: Ist ein Therapieverzicht gegen den Willen des Patienten ethisch begründbar? Ethik Med 2010;22:89-102. $\checkmark 9$ Winkler E, Hiddemann W, Marckmann G, et al.: Ethical assessment of life-prolonging treatment. Lancet Oncol 2011;12:720-722.

10 Khushf G et al.: The scope of organizational ethics. HEC Forum 1998;10:127-135.

11 Spencer E, Mills A, Rorty M, et al.: Organizational Ethics in Health Care. Oxford, Oxford University Press, 2000.

12 Zentrale Ethikkommission der Bundesärztekammer. Dtsch Arztebl 2006;103(24): A 1703-1707.

13 Fletscher JC, Siegler M: What are the goals of ethic consultation? A consensus statement. J Clin Ethics 1996;7:122-126.

14 Neitzke G, Dörries A, Simon A, Vommann J (Hrsg): Aufgaben und Modell von Klinischer Ethikberatung. Jahrbuch Ethik in der Klinik 2008:58-74.

15 Winkler EC: Sollte es ein favorisiertes Modell klinischer Ethikberatung für Krankenhäuser geben? - Erfahrungen aus den USA. Ethik Med 2009;21: 309-322.

16 Simon A: Qualitätssicherung und Evaluation von Ethikberatung; in Dörries A, Neitzke G, Simon A, Vollmann J (Hrsg): Klinische Ethikberatung. Ein Praxisbuch. Stuttgart, Kohlhammer, 2008, pp 167181.

17 Härter $M$ et al.: Partizipative Entscheidungsfindung in der stationären Versorgung. Forum Public Heath 2004;44:15.
18 Leighl NB, Butow PN, Tattersall MH: Treatment decision aids in advanced cancer: when the goal is not cure and the answer is not clear. $\mathrm{J}$ Clin Oncol 2004;22:1759-1762.

19 Härter M, Loh A, Spies C (Hrsg): Gemeinsam entscheiden - erfolgreich behandeln. Neue Wege für Ärzte und Patienten im Gesundheitswesen. Köln, Deutscher Ärzte-Verlag, 2005.

20 Vogel BA, Helmes AW, Hasenburg A: Concordance between patients' desired and actual decisionmaking roles in breast cancer care. Psychooncology 2008;17:182-189.

21 Vogel BA, Leonhart R, Helmes AW: Communication matters: the impact of communication and participation in decision making on breast cancer patients' depression and quality of life. Patient Edu Couns 2009;77: 391-397.

22 Coulter A: Partnership with patients: the pros and cons of shared clinical decision making. J Health Serv Res Policy 1997;2:112-121.

23 Stacey D et al.: Decision aids for people facing health treatment or screening decisions. Cochrane Database Syst Rev 2011;(10):CD001431.

24 Jaspers K: Philisophie, Band 1. Berlin, Springer, 1932, pp 121-129.

25 Weidmann B: Existentielle Kommunikation in der ärztlichen Therapie. Zum medizingeschichtlichen Kontext eines jaspersschen Ideals. Unveröffentlichtes Manuskript (42 Seiten). 2007, p 3. 